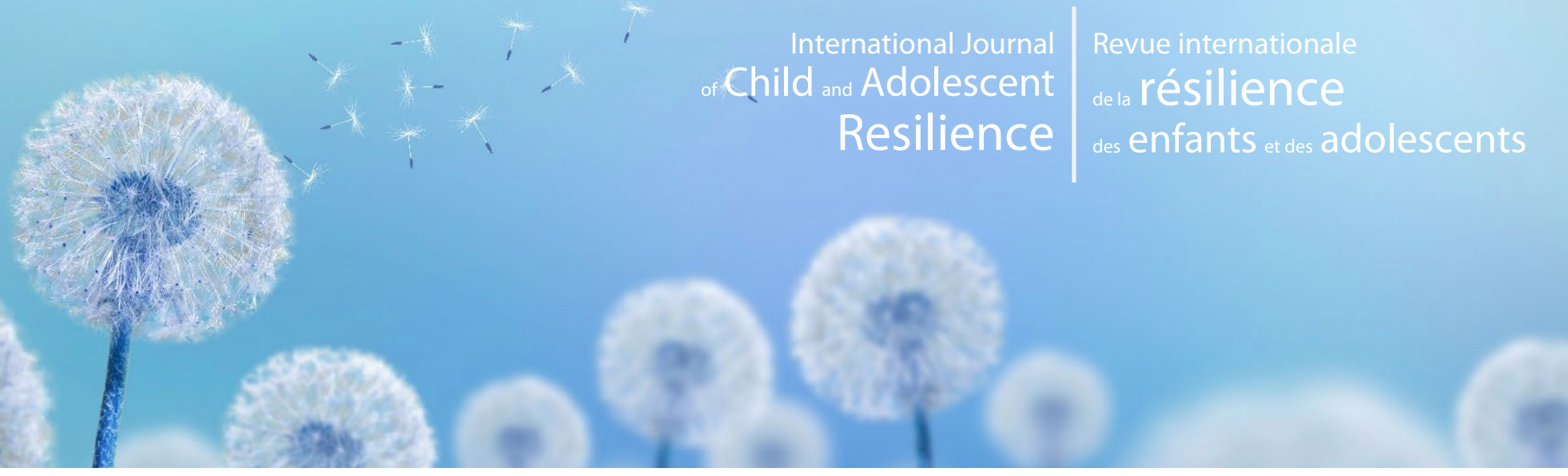

\title{
Comment soutenir la résilience des jeunes en transition vers l'âge adulte qui ont un parent atteint d'un trouble mental ? Ce qu'en pensent les jeunes concernés
}

\section{Aude VILLATTE ${ }^{1,2,3}$, Geneviève PICHÉ ${ }^{1,2,3}$ et Rima HABIB ${ }^{1,2}$}

\footnotetext{
1 Département de psychoéducation et de psychologie, Université du Québec en Outaouais

2 Laboratoire de recherche et d'actions pour les personnes ayant des problèmes de santé mentale et leurs proches (LaPProche)

3 Centre de recherche universitaire sur les jeunes et les familles (CRUJeF)
}

Auteur de correspondance : Aude Villatte, Ph.D, Département de psychoéducation et de psychologie, Université du Québec en Outaouais 5, rue Saint-Joseph. Saint-Jérôme (Qc), Téléphone : 1-800-567-1283 (poste \#4121).

Courriel : aude.villatte@uqo.ca

\section{Résumé}

Objectifs : Cette étude visait à : a) préciser le vécu des Jeunes ayant un Parent atteint d'un Trouble Mental (JPTM) au cours de leur transition vers l'âge adulte, en mettant en évidence tant les défis qu'ils rencontrent que les facteurs de protection leur permettant de composer avec ces défis; b) identifier des pistes de solution à envisager pour soutenir la résilience des JPTM transitant vers l'âge adulte.

Méthode : Les données ont été recueillies au cours d'ateliers Photovoice menés auprès de neuf participants (âge moyen : 22,5 ans). Cinq rencontres de deux heures ont permis à ces jeunes d'échanger autour de photographies au cours du printemps 2019. L'analyse de contenu des données retranscrites a été réalisée en collaboration avec les participants.

Résultats : Le fait d'avoir un parent atteint d'un trouble mental apporte son lot de défis lors de la transition vers l'âge adulte, en influençant notamment la prise d'autonomie, la construction identitaire et le parcours académique ou professionnel des jeunes. Un certain nombre de ressources individuelles, relationnelles et contextuelles sont identifiées, par les jeunes eux-mêmes, comme des facteurs leur permettant de composer avec ces défis. Des pistes de solutions impliquant différents milieux (scolaire, communautaire, santé et services sociaux) sont proposées pour soutenir la transition vers l'âge adulte des JPTM.

Implications: Des recommandations pratiques sont formulées sur la base des pistes de solutions proposées par les jeunes et des meilleures pratiques recensées dans la littérature.

Mots-clés : Photovoice; transition vers l'âge adulte; parent atteint d'un trouble mental; défis; facteurs de protection. 


\section{Introduction}

Le fait d'avoir un parent atteint d'un trouble mental constitue un facteur de vulnérabilité majeur, prédisposant aux problèmes de santé mentale et aux difficultés d'adaptation psychosociale et scolaire tout au long du développement. Durant l'enfance, les jeunes ayant un parent atteint d'un trouble mental (JPTM) démontrent davantage de problèmes émotionnels, d'hyperactivité et d'agressivité et plus de problèmes cognitifs (notamment langagiers), comparativement à leurs pairs de la population générale (Letourneau et al., 2006). À l'adolescence et au cours de la transition vers l'âge adulte, les JPTM sont plus à risque d'avoir des difficultés académiques et d'abandonner leurs études, de présenter des comportements délinquants, une consommation problématique de substances et des problèmes de santé mentale, comparativement à leurs pairs du même âge (Bergeron et al., 2000; Ellis et Hoskin, 2018; Ensminger et al., 2003; Jacobs et al., 2015; Ng-Knight et al., 2018; van Santvoort et al., 2015; Weissman et al., 2006). Le risque que ces jeunes développent de telles difficultés peut toutefois être réduit de moitié si des mesures de soutien et des interventions appropriées sont mises à leur disposition (Siegenthaler et al., 2012). Sachant que ces jeunes représentent au moins $20 \%$ de la population âgée de 0 à 25 ans (van Loon et al., 2015), et compte tenu des répercussions humaines et économiques considérables engendrées par les problèmes de santé mentale et les difficultés d'adaptation psychosociales (Weich et al., 2011), la nécessité de développer des mesures de prévention et d'intervention efficaces pour cette clientèle figure au cœur des politiques de santé publique (p. ex., Ministère de la Santé et des Services sociaux [MSSS] du Québec, 2015, 2017).

La prise en compte du savoir expérientiel des personnes susceptibles d'utiliser des services en santé mentale et de leurs proches constituerait une approche probante pour s'assurer de la pertinence et de la qualité des mesures de soutien qui leur sont destinées (MSSS, 2016a). À cet effet, quelques études ont questionné des JPTM à propos de leur vécu et de leurs besoins (p. ex., Bosch et al., 2017; Dam et Hall, 2016; Simpson-Adkins et Daiches, 2018; Yamamoto et Keogh, 2018). Celles-ci suggèrent que les JPTM rencontrent différents défis d'ordre familial, individuel et contextuel. La présence d'un parent atteint d'un trouble mental au sein d'une famille serait ainsi associée à : a) des relations parentsenfants moins positives, un risque accru d'abus, de négligence et d'exposition à de la violence conjugale au sein de la famille et une tendance à la parentification (c.à-d., inversion des rôles parent-enfant); b) des difficultés de régulation émotionnelle, un style d'attachement insécure, des émotions telles que la tristesse, la honte ou la culpabilité, et un manque d'habiletés sociales chez le jeune; c) une situation de précarité financière, des préjugés à l'égard des problèmes de santé mentale et un faible soutien social dans les différents milieux de vie du jeune et de sa famille. Différents facteurs permettant à ces jeunes de faire face à ces défis (autrement dit, d'être « résilients ») sont également identifiés dans ces études, par les JPTM eux-mêmes : stratégies de gestion du stress efficaces, présence d'un parent ayant une bonne santé mentale, compréhension du trouble parental, relations positives avec d'autres membres familiaux ( $p$. ex., fratrie, oncle/tante), participation à des activités de loisirs, accès à des ressources d'aide, etc.

Aussi intéressantes soient-elles, ces études ne permettent de comprendre précisément les enjeux ni les besoins spécifiques des JPTM au cours de leur transition vers l'âge adulte, aujourd'hui reconnue comme une période charnière du développement se déroulant approximativement entre 16 et 25 ans (Masten et al., 2004; Roberge et Déplanche, 2017; Schulenberg et al., 2004). En effet, celles-ci ont principalement été réalisées auprès d'enfants et d'adolescents (p. ex., Mordoch et Hall, 2008; Trondsen, 2012), auprès d'adultes rapportant leur expérience de façon rétrospective (p. ex., Metz et Jungbauer, 2021; Neziroglu et al., 2020; Reupert et al., 2019) ou encore auprès d'échantillons d'âges très variés sans tenir compte des spécificités développementales (p. ex., Bosch et al., 2017; Dam et Hall, 2016; Simpson-Adkins et Daiches, 2018; Yamamoto et Keogh, 2018). Considérant que la période de transition vers l'âge adulte comporte ses propres défis (p. ex., prise d'autonomie financière, résidentielle et affective, découverte des études postsecondaires ou du monde du travail, choix déterminants pour la future vie adulte et consolidation de son identité, développement de relations intimes et stables; Arnett, 2014; Burt et Paysnick, 2012; Luecken et Gress, 2010) et constitue une période aussi bien propice aux difficultés adaptatives (Institute of Medicine and National Research Council, 2015; Leipold et al., 2019) qu'aux trajectoires de résilience émergente (Goldstein et al., 2013; Grob et al., 2020; Masten et al., 2004), il convient d'explorer davantage le vécu et les besoins des JPTM au cours de cette période. Une meilleure compréhension des facteurs susceptibles de soutenir la résilience des individus à cet âge de la vie serait un atout majeur pour favoriser des trajectoires adultes épanouies (Masten et al., 2004; Schulenberg et al., 2004) et limiter ainsi les risques de transmission intergénérationnelle des problèmes de santé mentale (Institute of Medecine and National Research Council, 2015).

Quelques rares études ont sollicité des JPTM transitant vers l'âge adulte afin de comprendre les défis qu'ils rencontrent, les moyens qu'ils sollicitent pour faire face à ces défis et leurs besoins en termes de soutien (p. ex., Abraham et Stein, 2013; Ali et al., 2012; Brawer-Sherb et al., 2020; Kaimal et Beardslee, 2015; Patrick et al., 2019). Les participants 
de ces études disent rencontrer plusieurs difficultés, principalement: inquiétude et culpabilité à l'idée de quitter son parent, difficulté à établir des relations amoureuses satisfaisantes, prise de nombreuses responsabilités pour soutenir leur parent, difficultés à maintenir leur propre santé mentale, etc. Le soutien social (p. ex., d'amis, d'un partenaire) et la prise de distance (physique et/ou émotionnelle) avec le parent sont présentés comme des facteurs de protection cruciaux. Ces jeunes souhaiteraient, par ailleurs, recevoir davantage d'informations sur le trouble mental de leur parent, avoir accès à un réseau de JPTM et à un professionnel de façon anonyme (p. ex., via Internet) et considèrent que des actions devraient être menées pour réduire la stigmatisation entourant les troubles mentaux.

En plus d'être peu nombreuses et majoritairement réalisées à l'étranger, les études ayant considéré le point de vue des JPTM transitant vers l'âge adulte présentent un certain nombre de limites. La plupart se centrent davantage sur les difficultés rencontrées par ces jeunes que sur leurs capacités adaptatives et n'abordent que certains aspects de l'expérience de ces jeunes (p. ex., phénomène de parentification, transition à la parentalité) ou encore les défis rencontrés par des JPTM d'un groupe très particulier (p. ex., filles de mères monoparentales ayant un trouble dépressif). Notons par ailleurs que ces études adoptent toutes des devis de recherche «traditionnels » en interrogeant les jeunes à l'aide de questionnaires ou d'entrevues et en considérant ceux-ci comme des «sujets de recherche » plutôt que comme des « partenaires de recherche » qui auraient, par exemple, la possibilité de participer à l'analyse des données. L'intérêt de recourir à des méthodes de recherche participatives axées sur des médiums artistiques - telle que la méthode Photovoice qui s'appuie sur la photographie et la narration (Wang et Burris, 1997) - a pourtant été démontré auprès d'autres clientèles, notamment dans le champ de la santé mentale (Douville et al., 2017; Lorenz et Kolb, 2009; Marcotte et al., 2019). Le fait de participer à ce type de recherche stimulerait la réflexion et l'expression des participants tout en leur permettant de devenir plus conscients des enjeux récurrents qu'ils rencontrent, d'envisager des solutions qui font sens collectivement et d'avoir le sentiment de contribuer à un changement social en produisant des données qui seront portées à la connaissance des décideurs.

Cet article rend donc compte d'une étude menée à l'aide de la méthode Photovoice dans le but de mieux comprendre comment soutenir la transition vers l'âge adulte des JPTM. Les objectifs poursuivis étaient, plus précisément : a) de cerner le vécu des JPTM au cours de la transition vers l'âge adulte, en mettant en évidence tant les défis qu'ils rencontrent que les facteurs de protection leur permettant de composer avec ces défis; 2) d'identifier des pistes de solution à envisager pour soutenir la résilience des JPTM transitant vers l'âge adulte.

\section{Méthodologie}

\section{Recrutement et description des participants}

L'annonce de recrutement pour participer à des ateliers de groupe Photovoice a été diffusée sur les sites des réseaux sociaux de différents partenaires (p. ex., Réseau Avant de Craquer, Université du Québec en Outaouais, Cégeps des Laurentides et de l'Outaouais). Celle-ci précisait la nature du projet et les critères d'inclusion retenus : être âgés de 16 à 25 ans, avoir au moins un parent ayant un trouble mental (difficultés ayant affecté significativement le fonctionnement du parent au cours des 18 derniers mois, du point de vue du jeune), résider à proximité des lieux de collecte de données, parler et comprendre suffisamment le français et consentir librement à prendre part à ce projet. Cette étude a reçu l'approbation éthique du Comité d'éthique de la recherche de l'UQO (\#2019-191).

Sur les 199 jeunes ayant cliqué sur le lien contenu dans l'annonce de recrutement, 13 ont indiqué correspondre aux critères d'inclusion, ont consenti aux différentes clauses du formulaire de consentement et ont transmis leurs coordonnées afin de pouvoir être contactés par un membre de l'équipe de recherche. Une brève entrevue téléphonique a été réalisée avec chacun de ces jeunes afin de vérifier leur admissibilité, leur disponibilité et leur compréhension du déroulement des groupes Photovoice et des enjeux éthiques. Quatre jeunes se sont finalement retirés en raison d'un manque de disponibilité.

Au final, les neuf participants (huit femmes et un homme) sont tous majeurs (âge moyen $=22,5$ années). La plupart (78\%) occupent un emploi parallèlement à leurs études et la moitié (55\%) estime être dans une situation précaire. Tous ont une mère ayant un trouble mental (majoritairement un trouble de l'humeur, anxieux ou un trouble de la personnalité limite) et tous rapportent avoir des personnes de leur entourage proche qui présentent des problèmes de consommation. Moins de la moitié $(n=4)$ ont reçu un diagnostic de trouble mental, mais la plupart des participants disent présenter un certain nombre de symptômes anxieux ou dépressifs. 


\section{Collecte de données}

Les participants ont été répartis en deux groupes en fonction de leur lieu d'habitation (Laurentides ou Outaouais). Cinq rencontres de groupe hebdomadaires d'une durée de deux heures chacune s'échelonnant d'avril à juin 2019 ont été menées en suivant les principes de base de la méthode Photovoice. Deux facilitateurs (un homme et une femme), formés à la méthode et supervisés par l'équipe de recherche tout au long du projet, ont accompagné chacun des groupes en leur fournissant de l'information et en stimulant la participation et les discussions.

Lors de la rencontre d'introduction (rencontre 1), les participants ont été invités à envoyer aux facilitateurs, la veille de chaque future rencontre, les photos qu'ils souhaitaient présenter au groupe pour témoigner de leur vécu, accompagnées d'une légende et d'un titre récapitulant le message véhiculé par la photo. Au cours des trois rencontres suivantes, chaque jeune a eu l'occasion de présenter ses photos au groupe, dans l'ordre souhaité et à tour de rôle. Les rencontres 2 et 3 étaient principalement dédiées à l'exploration des défis rencontrés tandis que la rencontre 4 invitait les participants à évoquer les facteurs de protection mobilisés. Les membres du groupe pouvaient réagir aux photos de leurs collègues en nommant, par exemple, en quoi celles-ci faisaient écho à leur propre vécu. À la fin de chacune des séances, les participants étaient invités à réaliser une synthèse en identifiant les thèmes majeurs évoqués par le groupe, de façon visuelle (p. ex., schéma dessiné au tableau). Une photographie de chacune de ces synthèses a été prise par les facilitateurs. La dernière rencontre (rencontre 5) a permis de cibler les principaux besoins des JPTM sur la base des synthèses graphiques des séances 2 à 4, puis de réfléchir aux pistes de solutions pertinentes pour soutenir la transition vers l'âge adulte des JPTM.

\section{Stratégie d'analyse des données}

La chercheuse principale a procédé à une analyse de contenu des données retranscrites selon une méthode inductive. Cette analyse s'est appuyée sur les verbatim des rencontres, les légendes des photos et les synthèses graphiques faites par les participants. Au fil des lectures des données, des codes ont été attribués à chaque nouveau concept, puis regroupés en catégories pour créer un arbre thématique grâce au logiciel NVivo12. Après une révision de l'arbre thématique par l'équipe de recherche permettant d'aboutir à un accord interjuges, un résumé des résultats préliminaires a été présenté aux participants afin d'avoir leur rétroaction sur les sous-thèmes identifiés. Une dernière révision de l'arbre thématique a été réalisée à partir de leurs commentaires et une interprétation finale des résultats a été proposée.

\section{Résultats}

Cette section présente les thématiques et sous-thématiques évoquées au cours des rencontres Photovoice. Des photos, prises par les participants et accompagnées des légendes qu'ils ont rédigées, sont présentées pour illustrer les propos. L'ensemble des photos partagées lors des rencontres Photovoice, et dont les participants ont autorisé la diffusion, sont disponibles sur le site du LaPProche : https://lapproche.uqo.ca/exposition/.

\section{"C'est difficile de communiquer et souvent tendu, dans ma famille»}

Les participants évoquent unanimement les difficultés relationnelles qu'ils rencontrent avec leur parent atteint d'un trouble mental. À ce sujet, ils soulignent notamment les difficultés de communication engendrées par les symptômes parentaux:

Avec ma mère y'a toujours un fil rouge de manque de motivation et d'apathie... Y'a peu d'intérêt, peu d'émotion. Donc, c'est toujours difficile d'avoir une conversation. (Victoria) ${ }^{1}$

Plusieurs considèrent avoir une part de responsabilité dans ces difficultés, par exemple, en fuyant les échanges pour se protéger :

\section{Moi j'ai pas fêté la fête des Mères, j'ai pas appelé ma mère hier. Ça me fait de la peine, mais ça ne me fait pas du bien de l'appeler. (Joany)}

L'absence d'un regard valorisant, de limites claires à respecter et d'un soutien parental affectif, instrumental, mais aussi scolaire ou vocationnel est également rapportée :

Y'a jamais de félicitations. Ma mère était pas là non plus pour me dire : vas à l'école. J'peux facilement

\footnotetext{
${ }^{1}$ Certains participants ont souhaité conserver leur prénom; d'autres ont préféré en choisir un fictif.
} 
manquer des cours, elle me dit rien... (Claudia)

La relation avec le parent semble parfois même teintée de négligence et d'abus, tout particulièrement lorsque des problèmes de consommation de substances sont observés chez ce dernier :

Mon père y pète des coches comme ça là. I/ m'insulte, me traite de vache. (Coralie)

Au cours de leur transition vers l'âge adulte, plusieurs disent alors osciller entre la tentative de « faire le deuil » d'une relation positive avec leur parent et l'espoir toujours présent d'une amélioration. La photo 1 témoigne de plusieurs des enjeux vécus dans la relation avec le parent.

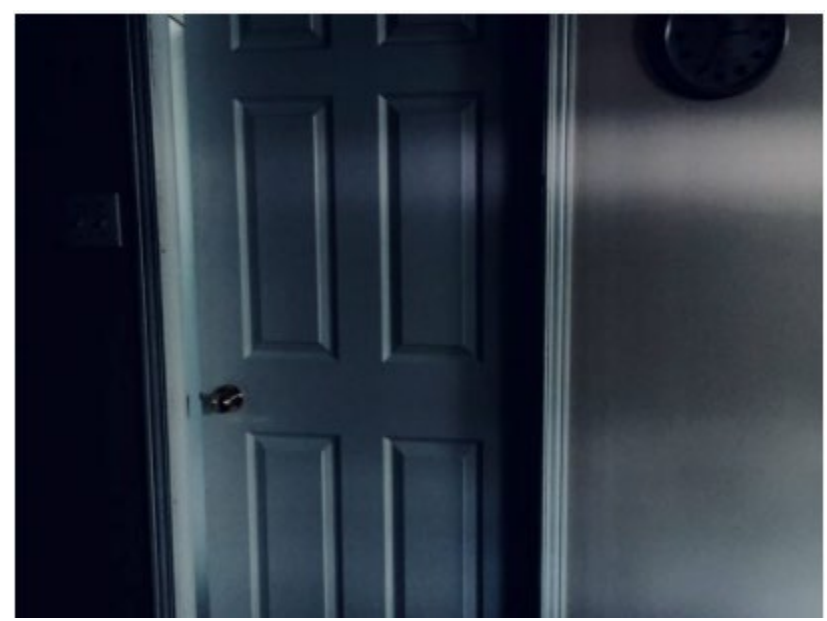

\section{Figure 1.}

\section{Fermeture}

Il est souvent difficile d'apporter du soutien à son parent, car c'est avoir l'impression d'être face à une porte qui reste presque toujours fermée : ton parent s'éloigne pour ne pas t'affecter et toi, tu t'éloignes pour te protéger. Mais malgré la distance et la fermeture, il y a plein d'espoir et plein d'amour! Les rayons de soleil et la porte entrouverte représentent les occasions où ma mère s'est confiée à moi et où j'ai pu l'écouter.

Victoria, 24 ans

Les relations familiales, au-delà des relations parent-jeune, semblent, elles aussi, être marquées par une absence de communication, un faible soutien et la présence de conflits et d'hostilité, voire de violence :

Mon père il a pas de dépression, mais c'est quelqu'un de très fermé qui considère que quand t'as un problème, tu fais avec pis tu prends ton trou. (Marianne)

Du fait de ces caractéristiques familiales, les jeunes disent avoir du mal à trouver un ancrage familial et à se sentir en sécurité dans leur propre famille:

T'as ton domicile, t'as ton chez-toi, mais tu te sens pas en sécurité. Pas tant chez toi. (Joany)

Certains participants soulignent toutefois qu'ils ont la chance d'entretenir des relations positives avec d'autres membres significatifs de leur famille (autre parent non atteint, fratrie, oncle ou tante) et de bénéficier d'un soutien (notamment émotionnel) de leur part :

T'sais mes oncles étaient là pis y m'encourageaient plus que ma mère. Pis quand ils me voyaient "félicitations, on est fiers de toi". (Bianca)

\section{"C'est comme si je devais choisir entre aider mon parent et construire ma propre vie »}

L'absence de soutien provenant de l'entourage familial contraste avec l'ampleur de celui que les jeunes disent apporter à leurs proches. L'ensemble des participants disent ainsi offrir un soutien affectif (p. ex., réconfort, écoute) et instrumental (p. ex., ménage, cuisine) important à leur parent et aux membres de leur famille (p. ex., soins apportés à la fratrie). Ils rapportent que la transition vers l'âge adulte a entrainé un accroissement de ces responsabilités : possibilité de véhiculer le parent, prêt d'argent à la famille, prise de décision concernant les soins, etc. Une inversion des rôles, ou parentification, peut alors avoir tendance à s'opérer entre le parent et le jeune. En attestent, par exemple, les propos de Daniel qui considère que c'est à lui de préparer ses parents à son départ du foyer parental, plutôt que l'inverse :

J'essaie de préparer mes parents que éventuellement je devrais partir... je sais pas pantoute comment ils vont réagir dans le fond alors je le fais progressivement.

Du fait du temps, de l'argent et de l'énergie consacrés au soutien familial, le jeune peut avoir du mal à répondre à ses propres besoins (p. ex., réfléchir à ce qu'il aimerait faire de sa vie, avoir le temps de voir ses amis) : 
Tu donnes beaucoup, mais à un moment donné, toi, ça t’enlève ton énergie, ça t'enlève ton temps pis ça fait que, toi, ça te rabaisse dans ton développement. (Marianne)

La poursuite d'études supérieures comme l'entrée sur le marché du travail peuvent notamment être compliquées par les enjeux de conciliation études/travail/famille comme par le manque de disponibilité cognitive et émotive que requiert l'investissement dans ces projets :

\begin{abstract}
Je pensais à l'université. Donc c'était des économies que je m'étais fait. Des projets que j'ai dû mettre de côté au moment où ma mère a fait sa dépression. Je l'ai aidée à payer l'appartement, l'épicerie et tout. (...) et puis c'est difficile de se concentrer sur tes études, étudier quand t'sais, t'es toujours à te demander "est-ce que je dois l'appeler? Est tu correcte? Est-ce que je dois aller la voir?». (Marie-Pier)
\end{abstract}

La figure suivante représente le choix que plusieurs estiment devoir faire entre le fait de soutenir leur parent et celui de construire leur propre vie d'adulte.

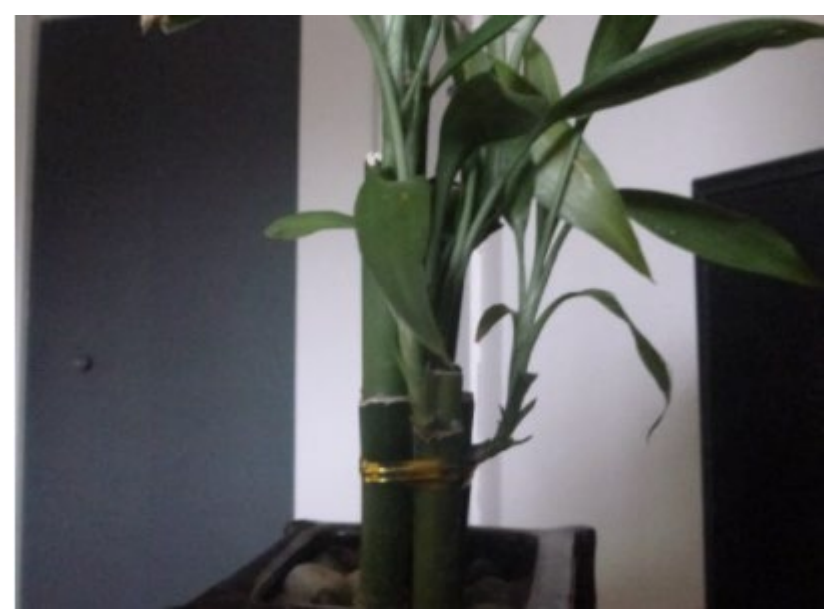

\title{
Figure 2
}

Le Choix d'une Vie

Devenir adulte quand on a un parent atteint d'un trouble mental, c'est faire un choix entre avancer soi-même ou faire avancer autrui au détriment de soi-même par amour. Lors de ma transition vers l'âge adulte, je me suis sentie divisée entre deux émotions principales soit, rester auprès de ma mère et continuer à m'occuper d'elle ou bien partir et me concentrer sur ma vie et mes projets. Cette photo démontre les différentes émotions, réflexions et choix que je n'aurais pas traversés si je n'avais pas eu un parent avec une maladie mentale.

Bianca, 22 ans

La prise d'autonomie résidentielle, soit le fait de quitter le foyer parental, peut également être « ralentie » par la crainte de ne plus être en mesure d'apporter toute l'aide nécessaire à son parent. Le fait de savoir son parent soutenu par des membres de l'entourage informel comme par des ressources professionnelles constitue alors un facteur de protection important. Plus largement, plusieurs participants évoquent que le fait de savoir leur famille à l'abri du besoin (financier, alimentaire, de protection) facilite leur centration sur leurs propres besoins :

Quand elle était en désintox, je savais qu'elle était avec des personnes qui sont là pour l'aider, faque moi, j'étais moins stressée de qu'est-ce qui va se passer ... faque c'était moins une préoccupation de quitter. Et puis maintenant, ça va mieux parce que ma mère elle a son logement, elle a plus la garde de ma soeur alors c'est moins une préoccupation de : Est-que c'est correct? Est-ce que ma soeur est correcte?(Marianne)

Dans les faits, le départ du foyer parental semble avoir un effet plutôt positif chez ceux qui ont fait le choix de partir :

Depuis que j'ai quitté, j'ai l'impression que ma vie est meilleure. Y'a plus de positif. J'ai une meilleure relation avec ma mère parce que je suis moins à devoir toujours m'occuper d'elle au quotidien. (Claudia) difficile :

Quand le participant a le sentiment d'être parti contre son gré et « précipitamment », l'expérience semble plus

Moij'ai pas eu le choix, il a fallu que je quitte vu que ma mère elle avait plus le goût d'être une mère. (MC)

« Je ne me connais pas tant que ça en tant que personne» 


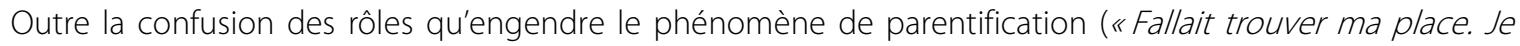
devais être le fils de mes parents, le frère de mes frères et leur parent. Même le mari pour ma mère parce que les problèmes financiers c'était avec moiqu'a parlait », Daniel), le peu de temps disponible pour se consacrer à l'exploration identitaire en raison d'une centration importante sur les besoins du parent complexifie la connaissance de soi. Les participants disent ainsi mieux connaitre leur parent qu'eux-mêmes, ce qui peut générer de l'anxiété à un âge auquel ils sont censés préciser qui ils sont et ce qu'ils souhaitent devenir :

J'ai pris conscience que je me connais pas tant que ça en tant que personne. C'est beaucoup plus facile pour moi de comprendre les autres vu que j'ai dû le faire avec mon père. Je suis un peu handicapé vis-à-vis de moi-même. Ça móbsède. (Daniel)

L'identité du jeune semble, en fait, très axée sur leur rôle d'aidant. Ils se décrivent comme « un outil de bien-être pour leur parent» (Marianne), disent se distinguer de la plupart des jeunes de leur âge par leur empathie, leur capacité d'écoute et leur habileté à comprendre autrui ( Je suis devenu un expert pour ça. Savoir comment mon parent se sent, dès que je rentre dans la pièce. Ses émotions étaient tellement instables faque fallait que je prenne tous les détails pour comprendre comment il allait», Daniel) et sont, pour la plupart, engagés dans des études/carrières en relation d'aide. S'ils considèrent que cela s'inscrit logiquement dans leur parcours de vie, certains se questionnent tout de même sur les risques que cela comporte, notamment en termes d'épuisement professionnel :

Moi j'ai trouvé un intérêt là-dedans (criminologie). C'est pas un centre d'intérêt, c'est quelque chose qui fait partie de moi. Je suis née là-dedans, avec les problèmes de mon père, pi ça va me suivre toute ma vie faque aussi bien d'aller dans quelque chose que je connais déjà... Après, est-ce que c'est une bonne clientèle pour moi? Je ne sais pas. (Coralie)

L'absence de modèles adultes positifs auxquels s'identifier et une histoire familiale marquée par l'instabilité compliquent la projection de soi dans un avenir positif et stable :

Devenir adulte quand on a un parent atteint d'un trouble mental, ça peut amener à se sentir petit et peu en confiance en raison de l'absence de modèles adultes positifs. Peut-être que nos modèles avaient eux-mêmes de la difficulté à s'accrocher à la vie en raison de leur difficulté. Alors, on ne se sent pas équipé pour faire face à ce monde vaste et on s'imagine encore petit. (Victoria)

La peur de développer les mêmes troubles que son parent colore la façon dont le jeune anticipe son avenir. L'apparition de symptômes anxieux ou dépressifs ou les opportunités de prise de risque qu'occasionne la période de

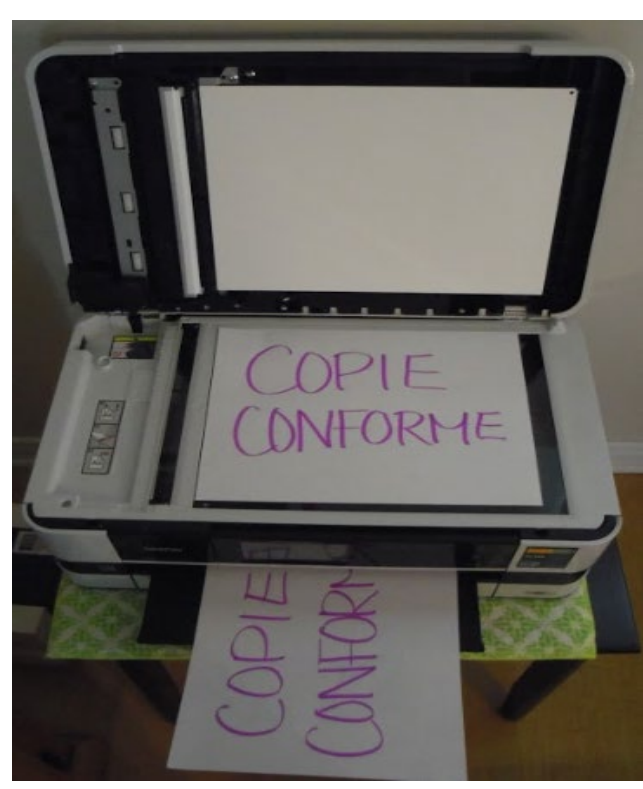
transition vers l'âge adulte ont tendance à renforcer ces craintes, comme en témoigne la photo suivante.

\section{Figure 3.}

\section{Copie Conforme}

J'ai la peur de devenir la copie conforme de ma mère, de reproduire ses comportements et ses dépendances. C'est une peur qui me hante constamment dans une période où on cherche à se définir par soimême en tant qu'individu autonome et indépendant, et où on veut vivre nos expériences et essayer l'alcool, certaines drogues, etc.

Marie-Pier, 21 ans 
Malgré les défis qu'elle engendre, la transition vers l'âge adulte peut être synonyme de « renaissance » par la possibilité qu'elle offre de s'affranchir de l'histoire familiale et d'entreprendre ses propres projets:

Je me sens revivre. Maintenant, j'ai la possibilité de créer mes propres racines pour apprendre à m'épanouir et fleurir grâce à mes propres succès! (Joany)

L'investissement dans un projet (p. ex., scolaire, artistique, sportif), l'identification d'objectifs à atteindre, l'engagement dans des relations stables comme le fait de trouver d'autres modèles adultes inspirants dans leur entourage agissent, alors, comme des moteurs puissants :

Ben je me suis mis des objectifs tout au long de ma vie, pis tout au long je les ai respectés. Ma priorité c'était l'école pi je me concentrais là-dessus. Maintenant c'est trouver une job stable. Je voulais juste comme ... pas avoir la vie que j'ai eue. Faque moi, ça máa juste motivée. Offrir la meilleure vie que je peux et avoir tout ce que j'ai pas pu avoir chez ma mère ... pouvoir le donner à mes enfants plus tard. (Bianca)

Ces derniers propos attestent du fait que la projection de soi en tant que parent peut être porteuse d'espoir. Notons toutefois que deux participants rapportent une ambivalence importante à l'idée d'avoir des enfants :

Les problèmes de santé mentale ça peut arriver à n'importe qui pis je me dis, je veux tu prendre la chance que moi je dérape à un moment donné pis que mon enfant vive ça? Pis d'un autre côté je me dis : ben peut-être que j'aimerais ça être mère, peut-être quej'aimerais ça créer la relation que j'ai pas réussi à maintenir avec ma mère. Mais je suis encore dans l'ambivalence... est-ce que je prends ce risque-là ou je laisse faire pis mes projets de vie ça sera autre chose?(MC)

Les difficultés engendrées par le trouble mental du parent et la stigmatisation qui entoure celui-ci peuvent impacter la confiance en soi du jeune et entrainer un phénomène d'autostigmatisation :

Je me sens niaiseuse, incompétente, à cause de ma mère. À me faire dire que j'suis pas normale à cause de ma mère. Ma mère elle fait rien dans la vie pis moi la pire insulte que tu peux me dire dans vie c'est que tu ressembles à ta mère. (Joany)

Parce que quand tu vois ta mère qui veut pu être une mère, tu te dis : Qu'est-ce que j'ai fait en tant qu'enfant pour que ma mère ait pu le goût d'être une mère?(MC)

D'autres jeunes s'estiment fiers de ce qu'ils ont su traverser à travers leur expérience familiale et des qualités dont ils ont fait preuve pour cela : force, persévérance, maturité, autodiscipline. Leur estime d'eux-mêmes s'en trouve alors renforcée :

Du fait de mon parcours, j'ai toujours été plus mon propre modèle. Ç'a m’a aidé de comme, je sais que j'ai une force à l'intérieur de moi, pis la plupart des gens l'ont pas. (Daniel)

\section{«Un tourbillon d'émotions et de préoccupations »}

Les jeunes se disent traversés par un grand nombre d'émotions (p. ex., inquiétude, tristesse, honte, colère, sentiment d'impuissance et d'isolement, culpabilité) plus ou moins difficiles à assumer :

Je trouve ça difficile à vivre parce que moi, j'ai jamais été, de toute ma vie, fâchée contre ma mère, j'ai jamais été capable d'être fâchée contre elle ... Pis récemment j'ai commencé à être fâchée contre elle pis ça me met vraiment à l'envers. (MC)

La gestion de ces émotions semble d'autant plus ardue que le trouble mental parental a pu inhiber le développement socioaffectif du jeune, plus précisément ses capacités à identifier, exprimer et réguler ses émotions :

C'est difficile de comprendre mes émotions, de les gérer, du fait que ma mère maurait pas montré comment faire ... parce qu'elle le fait pas elle-même. (Marianne)

L'impression «que tout ne tient qu'à un fil », qu'il s'agisse de la santé mentale de leur parent, de leur propre santé et sécurité, et du cours des choses en général, semble omniprésente chez les participants et teinter leur univers émotionnel, comme en témoigne la photo de Daniel (figure 4) et les propos suivants :

L'instabilité familiale, ça fait que toi-même ça te fait vaciller, comme un arbre dans la tempête. Tu sais 
jamais si ton arbre va se déraciner. Toujours se demander est-ce que le vent va être tellement fort que ça va complètement l'arracher ou tu vas avoir la force de rester. (Marianne)

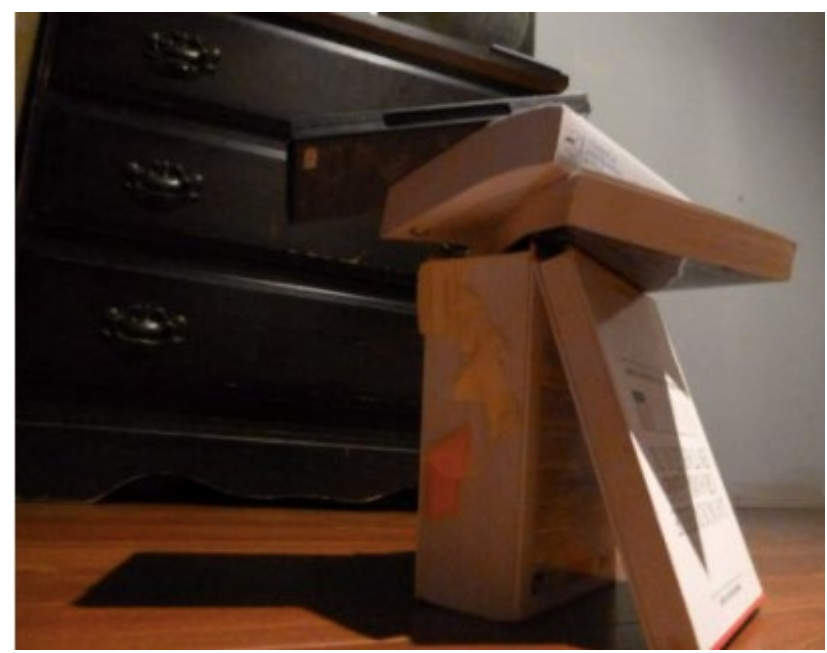

\section{Figure 4.}

Attention

Être un proche aidant pour son parent atteint d'un trouble mental, c'est être comme cette pile de livres qui semble se tenir, mais qui est prête à tomber à tout moment. J'aime considérer que comme proche aidant j'apporte de la stabilité chez moi. Cependant, je suis aussi conscient que tout peut basculer à chaque instant.

Daniel, 23 ans

Plus largement, différents types de croyances, modelées au fil de leurs expériences de vie, semblent entretenir leurs émotions et inquiétudes. Parmi celles-ci, notons le sentiment d'être responsable de la santé mentale de leur parent et d'être à l'origine de ses difficultés, une impression d'unicité (c.-à-d., d'être le seul à vivre ce genre de défis), un mode de pensée en tout ou rien ou encore une tendance au catastrophisme :

Moice que je me suis dit c'est : est-ce que je reste pis je m'occupe de ma mère toute ma vie ou estce que je m'en vais faire mes projets pis j'avance dans ma vie et je la laisse toute seule. Pis t'sais, mettons si je l'avais comme laissée tomber, ça aurait été comment? Elle va tu mourir? Tu te sens coincée. (Bianca)

L'absence de connaissances adéquates concernant le trouble mental parental et de communication sociale et familiale autour des problèmes de santé mentale entretiendrait ces croyances:

J'avais pas encore de connaissances, je savais pas c'était quoi le problème. J'étais comme "si je suis parfaite, ça va aider ma mère pis ça va disparaitre, t'sais ... y'en aura pu de problèmes ... Qu'est-ce que je peux faire pour devenir une meilleure personne? Si moi j'agis bien, j'agis de la bonne façon, je l'aide de la bonne façon, ben ça va disparaitre! Ça va s'en aller, toute va s'arranger comme par magie. » Et puis tu n'as pas l'impression qu'ily a d'autres mondes qui vivent ça vu que personne n'en parle. Faque on se sent vraiment seul. (Marianne)

La possibilité d'acquérir des connaissances à propos des troubles mentaux et certaines prises de conscience permettent, à l'inverse, de rétablir un mode de pensée plus fonctionnel et d'apaiser les émotions et inquiétudes des participants :

À force, j'ai compris qu'on ne peut pas aider quelqu'un qui ne veut pas s'aider lui-même. II faut apprendre à comprendre qu'on ne peut pas faire plus que ce que la personne veut. (Bianca)

Un mécanisme de «contagion émotionnelle » semble, enfin, s'opérer entre le parent et le jeune :

Si ma mère était de bonne humeur, ben c'était une bonne journée pour moi aussi. Si je revenais de travailler pis elle était pas de bonne humeur, ben ça brisait ma journée. (Victoria)

Pour s'en protéger, l'ensemble des participants souligne l'importance de mettre ses limites et la plupart identifient des stratégies qu'ils déploient pour cela :

Des fois, ma mère elle fait juste comme chialer, moi je fais juste mettre mes écouteurs. Pis avec ma musique, ça me ramène à la réalité, à mon équilibre émotionnel. (Marie-Pier).

Ils témoignent toutefois d'une certaine ambivalence à ce sujet en raison des coûts associés à cette mise à distance : 
J'ai essayé avec toute la douceur qui m'habite de lui dire que je voulais plus qu'elle m'appelle quand elle avait consommé. Pis là, je pense qu'elle s'est sentie vraiment mal, elle a pleuré. À partir de ce moment-là, y'a comme quelque chose qui s'est brisé. Elle máppelait moins ... elle voulait pas être un fardeau pour moi faque elle a pris ses distances. Pis ça, ça m'a fait vraiment mal. Parce que tout le monde me dit à quel point c'est important de mettre ses limites, de s'écouter, pis là moi je l'ai fait pis ça a vraiment mal viré. Je m'en suis voulu vraiment d'avoir fait ça. (MC)

«Maintenir sa propre santé mentale : tout un défi ! »

L'ensemble des défis évoqués jusqu'à présent mettent la santé mentale du jeune, mais aussi sa santé physique, à rude épreuve :

Vraiment, ma santé mentale en prenait un coup. J'étais tellement tout le temps anxieuse que même ma santé physique ... ça commençait à se ressentir. T'sais d'avoir de la misère à respirer ou d'être pris toujours une boule dans la gorge comme si ça allait déchirer. (MC)

Outre le fait de «mettre leurs limites», les participants disent recourir à plusieurs stratégies pour tenter de préserver leur santé mentale: utiliser l'humour, "ne pas penser aux problèmes», "voir les choses positivement», «s'étourdir dans l'action» ou passer du temps avec des amis, par exemple. La pleine conscience et la pratique d'activités sportives et artistiques sont rapportées comme des stratégies efficaces à plusieurs égards. Celles-ci permettraient, selon les jeunes, d'évacuer un trop-plein d'émotions, d'avoir un répit, d'améliorer leurs habiletés socioaffectives (p. ex., régulation émotionnelle, confiance en soi), mais aussi de rétablir un mode de pensée plus fonctionnel :

Quand j'arrive à ces points de vue, ça permet de voir les choses sous un autre angle, ma vie c'est pas juste les problèmes avec mon parent. (Joany)

Si plusieurs considèrent que le soutien social informel ou formel peut également aider à maintenir leur santé mentale, voire s'avérer nécessaire ("Des fois, t'es pas la meilleure personne pour t'aider. Faut que t'ailles chercher de l'aide à l'extérieur. Moi ça m'a beaucoup aidée», Claudia), ils évoquent leurs difficultés à demander de l'aide. Pour ceux qui ont la chance d'entretenir des relations positives avec certains membres de leur famille, la peur de peser sur des personnes déjà éreintées par le trouble mental parental serait un frein important :

Je peux pas leur demander de máaider (à mes frères), y'ont tellement dépensé d'énergie que, moi, ça serait la goutte qui ferait déborder le vase. (Daniel)

La demande d'aide auprès de l'entourage non familial ne semble pas non plus évidente. Confrontés à des préjugés à l'égard des personnes ayant des problèmes de santé mentale ou de leurs proches comme à des remarques désobligeantes ou un manque de considération au moment d'évoquer le sujet de la santé mentale, les participants ne se sentent pas toujours à l'aise de partager leur vécu:

Dans mon entourage, c'est comme ça qu'on le perçoit: "Ah quand tu parles de ta mère ... Ah change donc de sujet... ", faque j'ai jamais la possibilité d'en parler. Toujours l'impression de les mettre mal à l'aise. (Joany)

Quant aux obstacles nuisant aux demandes d'aide formelle, les participants évoquent la crainte d'être jugé ou de nuire au système familial ou encore la difficulté à identifier les personnes et ressources susceptibles de leur venir en aide :

T'as peur d'aller les voir, de leur dire ce que tu vis, pis qu'après ça y'appellent la DPJ pis que tu t'en ailles. Ça finit que tu vas voir personne. (Bianca)

T'as pas envie que les autres élèves te voient sortir du bureau de la TES, faque qui savent que y'a quelque chose qui marche pas chez toi ou dans ta famille. (Joany)

Quoi qu'il en soit, les jeunes soulignent combien le maintien d'une bonne santé mentale constitue un défi pour les JPTM et résulte d'efforts importants de leur part, comme en témoigne la photo de Claudia. 


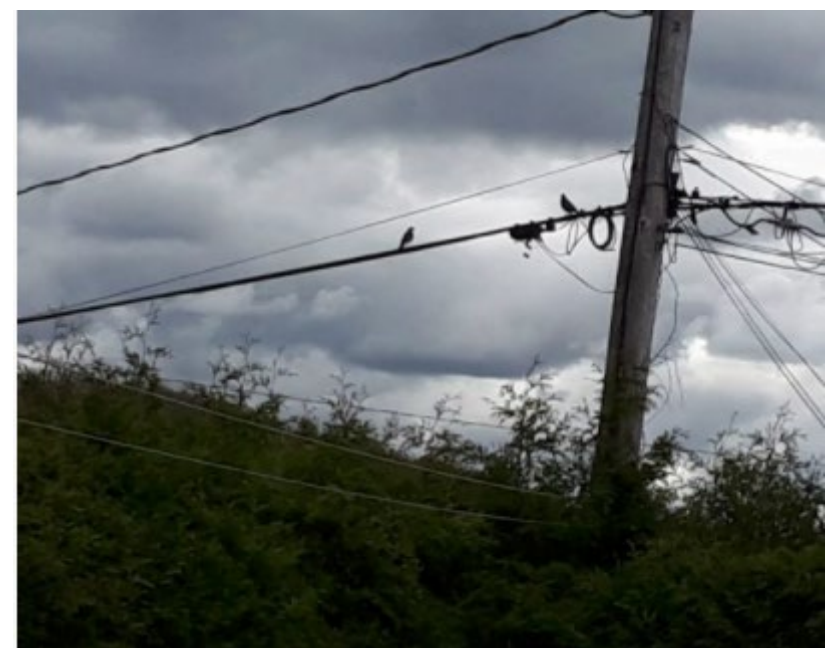

\section{Figure 5 .}

\section{L'équilibre}

Etre un proche aidant pour son parent atteint d'un trouble mental, c'est être comme ces deux oiseaux qui se tiennent proches et qui se regardent pour s'accrocher à la vie. J'ai habité avec ma mère durant mon adolescence et début de l'âge adulte et j'étais près d'elle pour la soutenir. À un moment donné, je vivais les mêmes choses et j'avais les mêmes problèmes sur les épaules qu'elle. J'ai vite appris à être solide et à appuyer ma mère. J'avais le rôle de la mère et j'étais mature. Elle s'accrochait à moi et j'essayais aussi de tout faire pour m'accrocher : on s'accrochait à la vie ensemble, comme ces oiseaux sur la même ligne.

\section{Claudia, 24 ans}

\section{« II y a des solutions pour permettre aux JPTM transitant vers l'âge adulte d'aller bien »}

Sur la base des défis et facteurs de protection identifiés, les participants ont proposé différentes pistes de solutions qui pourraient être mises en place pour soutenir la transition vers l'âge adulte des JPTM. Les pistes de solutions proposées prévoient des actions à différents niveaux et d'intensité variable pour être en mesure de soutenir le potentiel de résilience de ces jeunes.

À l'échelle sociétale, les participants considèrent qu'il est crucial de reconnaître les défis et besoins des JPTM au sein de la Politique nationale pour les proches aidants, en prévoyant notamment un soutien financier à destination de ceux qui font face à des défis de conciliation études/travail/famille. Ils soulignent l'importance de poursuivre les efforts en matière de : a) lutte contre l'intimidation et la stigmatisation à l'égard des personnes ayant des troubles mentaux et leurs proches et; $b$ ) de promotion de la santé mentale et de sensibilisation aux troubles mentaux. Dans ce cadre, les participants suggèrent de miser sur le numérique et d'impliquer des JPTM comme «porte-parole ». La stratégie voulant que certaines personnalités appréciées des jeunes puissent évoquer leur statut de JPTM publiquement (p. ex., \#JPTM sur Instagram) serait particulièrement gagnante, selon les participants.

Un soutien devrait être proposé aux JPTM et à leurs parents et les services en santé mentale devraient adopter une approche centrée sur la famille (p. ex., en considérant systématiquement les besoins des enfants de parents atteints d'un trouble mental). Outre l'importance d'assurer l'accessibilité des services (p. ex., en les offrant en ligne et dans les milieux fréquentés au quotidien par les jeunes) et leur visibilité, notamment via une promotion attrayante sur les réseaux sociaux, il s'agit de prévoir des mesures de soutien variées, susceptibles de répondre à différents besoins: soutien financier ou logistique (p. ex., livraison de repas ou aide-ménagère à domicile), informations (p. ex., sur les troubles mentaux, les ressources disponibles, etc.), activités sportives en groupe réservées à des JPTM, lignes d'écoute, répit, enseignement de stratégies de gestion de stress et d'habiletés de communication, thérapie individuelle ou familiale, etc. Pour ce faire, davantage de liens entre les services cliniques et communautaires sont souhaités.

Des professionnels et intervenants sensibilisés aux réalités des JPTM (c.-̀̀-d., en général et au cours de la transition vers l'âge adulte en particulier), mais aussi à l'importance de la santé mentale devraient être présents dans les milieux de vie fréquentés par les jeunes. Des actions et des mesures à l'échelle locale offertes à l'ensemble des jeunes (p. ex., journée de promotion de la santé mentale offerte dans un établissement scolaire), comme aux JPTM en particulier (p. ex., accommodements particuliers prévus dans la politique d'un établissement), sont recommandées. L'importance d'appliquer ces recommandations dans les milieux fréquentés par les jeunes de 16 à 25 ans comme dans ceux fréquentés par les plus jeunes est également soulignée, dans une optique de promotion/prévention.

L'ensemble de ces solutions, combinées, devrait permettre aux JPTM d'évoluer dans des contextes exempts de discrimination et de stigmatisation, d'avoir accès - pour eux et leurs parents - à du soutien matériel et psychosocial, d'avoir suffisamment d'habiletés et de ressources personnelles pour être en mesure de relever les défis rencontrés et de pouvoir demander de l'aide lorsque nécessaire. 


\section{Discussion et implications}

Malgré le nombre croissant de recherches étudiant l'influence des troubles mentaux parentaux sur les enfants à différentes périodes développementales, il existe très peu d'études qui examinent directement l'expérience de JPTM en transition vers l'âge adulte. Dans un contexte où les experts appellent à mieux saisir ce qui permettrait de soutenir le développement positif des jeunes en transition vers l'âge adulte en général - et des plus vulnérables en particulier - (MSSS, 2015; Roberge et Déplanche, 2017; Secrétariat à la Jeunesse, 2016), cette étude visait à préciser les défis, ressources et besoins des JPTM âgés de 16 à 25 ans.

Les résultats de notre étude rejoignent tout d'abord les défis identifiés chez les JPTM de différentes tranches d'âge en mettant notamment en évidence des difficultés de communication familiale, une tendance à la parentification, des émotions et préoccupations variées, des préjudices liés à la stigmatisation des troubles mentaux et un faible soutien social (Bosch et al., 2017; Dam et Hall, 2016; Simpson-Adkins et Daiches, 2018; Yamamoto et Keogh, 2018). De même, plusieurs facteurs déjà identifiés dans les études précédemment menées auprès de JPTM de différentes tranches d'âge semblent jouer un rôle protecteur important au cours de la transition vers l'âge adulte ( $p$. ex., stratégies de régulation émotionnelle efficaces, relations positives avec d'autres membres de la famille, réseau de soutien social de qualité) et contribueraient à expliquer qu'un certain nombre de JPTM transitant vers l'âge adulte vont bien malgré l'adversité à laquelle ils sont confrontés (Collishaw et al., 2016; Lewandowski et al., 2014; Pargas et al., 2010).

Les participants de cette étude soulignent, davantage que ne le laissent penser les résultats des études antérieures, l'influence qu'ils peuvent avoir sur les caractéristiques de leur milieu familial et social. Plusieurs soulignent ainsi combien leurs stratégies de protection peuvent contribuer aux difficultés de communication familiale ou à quel point ils choisissent d'apporter du soutien au parent pour éviter de trop s'inquiéter ou de culpabiliser. Ces résultats confirment l'importance de s'appuyer sur des modèles multifactoriels et transactionnels (voir par exemple celui de Van Doesum et al., 2005) pour comprendre et prévenir les phénomènes de transmission intergénérationnelle des problèmes de santé mentale.

Cette étude apporte, en outre, un éclairage plus précis sur ce qui se joue pour les JPTM au cours de leur transition vers l'âge adulte. Si elle confirme différents enjeux relevés dans les quelques études menées antérieurement auprès de JPTM de cette tranche d'âge spécifiquement (p. ex., difficultés d'engagement professionnel ou académique; Brawer-Sharb et al., 2020; Patrick et al., 2019), elle documente davantage les enjeux identitaires et ceux entourant la prise d'autonomie chez ces jeunes. Tandis que cette période développementale est habituellement considérée comme propice à la centration sur soi et à l'exploration identitaire (Arnett, 2014; Burt et Paysnick, 2012), il semble que les responsabilités endossées par les JPTM, leurs préoccupations à l'égard du parent et leur tendance à considérer les besoins de l'autre avant les leurs ne leur offrent pas l'espace suffisant pour se questionner pleinement sur qui ils sont et ce qu'ils souhaitent devenir. Leurs projets d'avenir sont principalement axés sur leur rôle de proche aidant et sur la recherche d'une stabilité qui leur a manquée, laissant davantage penser à un processus de forclusion identitaire (c.àd., une identité prédéterminée ou prescrite) qu'à une identité en voie d'achèvement, c'est-à-dire une identité réalisée une fois que l'individu a questionné ses possibilités et pris une décision en fonction de ce qui lui convient le mieux (Cohen-Scali et Guichard, 2008). La difficulté à intégrer leur histoire familiale et à se construire sans la rejeter ni fusionner avec elle semble également être au cœur des défis à relever par ces jeunes pour être en mesure d'aborder leur vie d'adulte et éventuellement, leur propre statut parental. Si, comme pour d'autres catégories de jeunes considérés «vulnérables » (p. ex., jeunes issus des services de la protection de la jeunesse, jeunes de la rue), la prise en charge de responsabilités semble se faire de façon précipitée, d'autres pans du processus d'autonomisation (p. ex., résidentiel, émotionnel) semblent quant à eux « ralentis » chez les JPTM, du fait des craintes à l'égard de la santé du parent, de la culpabilité ressentie à l'idée de le laisser, etc. Ces jeunes pourraient alors se sentir investis d'une responsabilité supplémentaire au seuil de leur vie adulte, qui se pose peut-être moins à d'autres catégories de jeunes dits «vulnérables » : celle de préparer leur.s parent.s à cette prise de distance.

Plusieurs résultats confirment que loin d'être une période uniquement porteuse de vulnérabilités, la transition vers l'âge adulte est également propice à la résilience. Au-delà du caractère exigeant et stressant des défis à relever, la possibilité de développer de nouveaux liens sociaux et intimes, la prise d'indépendance du milieu familial et l'engagement dans des études postsecondaires ou dans un emploi plaisant, seraient des facteurs de protection clés à cet âge de la vie, comme cela a déjà été observé auprès d'autres catégories de jeunes dits « vulnérables » (Madewell et Ponce-Garcia, 2016; Marcotte et al., 2014; Masten et al., 2006). Alors que chez les jeunes en transition vers l'âge adulte en général, les habiletés sociales telles que l'empathie, l'écoute et l'entraide comptent également parmi les principaux 
facteurs de résilience (Masten et al., 2004, 2006; Pauzé et al., 2019), il se pourrait que la capacité à mettre ses limites et à prioriser ses propres besoins soit davantage protectrice chez des jeunes qui rapportent une tendance à surinvestir le don de soi et l'aide à autrui.

Plusieurs pistes de solution, destinées à soutenir la résilience des JPTM au cours de leur transition vers l'âge adulte, ont été proposées par les participants. Elles concordent avec les pratiques adoptées par les pays chefs de file en matière de considération des JPTM (p. ex., pyramide des soins familiaux; Mottaghipour et Bickerton, 2005 ou EASE model; Foster et al., 2019), comme avec celles qui prévalent dans le champ de l'intervention auprès des jeunes en transition vers l'âge adulte en général ( $p$. ex., approche coordonnée, multimodale, intersectorielle et multidisciplinaire et promotion de la santé mentale au-delà de la prévention des troubles mentaux; Roberge et Déplanche, 2017). Si quelques initiatives à l'égard des JPTM sont déployées au Québec et au Canada, elles n'en sont qu'à leurs prémisses et concernent davantage les enfants et les adolescents (Piché et al., 2019). Les résultats de cette étude viennent conforter la pertinence de poursuivre le déploiement de ces initiatives et d'adapter les solutions en fonction des besoins spécifiques des jeunes de différentes tranches d'âge.

Des actions au niveau sociétal, comme par exemple la poursuite des campagnes de sensibilisation à l'égard des troubles mentaux et de promotion de la santé mentale permettraient de favoriser l'estime de soi et l'intégration sociale des JPTM et de leurs parents, faciliteraient leur demande d'aide et soutiendraient leur santé mentale et la résilience familiale (MSSS, 2016b). Au niveau local, les milieux de vie fréquentés par les jeunes devraient contribuer à ces efforts de sensibilisation et de promotion, dès le primaire et le secondaire, dans une optique de prévention et de promotion universelle. Comme le suggèrent les bonnes pratiques auprès d'une clientèle en transition vers l'âge adulte, il semble nécessaire d'impliquer des JPTM de 16 à 25 ans dans les actions de sensibilisation qui leur sont spécifiquement destinées afin d'être en mesure de toucher le public visé, de limiter l'autostigmatisation et d'offrir aux jeunes des modèles auxquels s'identifier (Roberge et Déplanche, 2017). La reconnaissance des JPTM au sein de la Politique nationale pour les proches aidants, qui mettrait en évidence les défis spécifiques qui se posent aux « jeunes aidants » et prévoirait des mesures adaptées, serait également fort utile. Un mémoire en ce sens a été déposé par l'équipe de recherche à la Commission des relations avec les citoyens lors des consultations particulières sur le Projet de loi no 56 (Villatte et al., 2020). Enfin, bien que non explicitement nommées par les participants, les politiques sociales de soutien aux familles et à l'insertion sociale, scolaire et professionnelle des jeunes à l'échelle nationale permettant aux parents comme aux jeunes de disposer de conditions de vie et de santé satisfaisantes semblent essentielles. Si celles-ci sont recommandées pour soutenir la santé mentale de l'ensemble des individus en transition vers l'âge adulte (Roberge et Déplanche, 2017), elles pourraient s'avérer particulièrement salvatrices pour des jeunes qui ont tendance à mettre leurs besoins de côté pour subvenir à ceux de leurs parents.

Différentes mesures d'aide et de soutien devraient être proposées aux JPTM transitant vers l'âge adulte par les milieux de la santé et des services sociaux, les organismes communautaires et les établissements scolaires. Au-delà des informations, du soutien et des stratégies qui seraient pertinentes à fournir aux JPTM de tout âge (p. ex., groupe de soutien, stratégies de régulation émotionnelle, compétences en gestion de conflit, en résolution de problèmes et en communication), des mesures destinées à favoriser notamment la prise d'autonomie, la construction identitaire et la recherche d'aide seraient probablement particulièrement bénéfiques auprès des JPTM en transition vers l'âge adulte (p. ex., aide financière et logistique, travail sur les craintes et croyances inhibant la prise d'autonomie et la demande d'aide, mise à contribution des JPTM dans des projets par et pour, services d'orientation scolaire et professionnelle). Pour les JPTM de cette tranche d'âge, le recours au numérique semble également incontournable. Cette génération, considérée comme celle des «natifs numériques», aurait en effet recours à Internet comme source privilégiée d'information (Oulasvirta et al., 2012) et comme moyen de communication (Collin et al., 2011). Plus largement, il est démontré que l'utilisation du numérique constituerait un facteur puissant du développement social et identitaire des jeunes en transition vers l'âge adulte (Gray et al., 2005). Sur la base de ces différents constats, un guide numérique a été développé par l'équipe de recherche et les participants à destination des JPTM de 12 à 25 ans du Québec. Celui-ci aborde l'ensemble des défis identifiés dans le cadre de cette étude, est disponible gratuitement en ligne sur le site du LaPProche (https://lapproche.uqo.ca/) et a été relayé par le Réseau Avant de Craquer à l'ensemble de ses partenaires, notamment aux établissements scolaires. L'évaluation de la satisfaction et des retombées de ce guide est actuellement en cours auprès d'un échantillon de JPTM, de parents et d'intervenants québécois.

Il apparaît enfin évident qu'il convient de ne pas se limiter à proposer des services et du soutien aux JPTM et d'adopter plutôt une approche familiale. Ici encore, un soutien et des interventions d'intensité variable sont recommandés. Si pour certaines familles, il s'agirait de référer les parents et leurs enfants à des ressources d'aide ou de 
soutien général (p. ex., lignes d'écoute), de fournir de l'information (p. ex., sur l'impact du trouble mental sur la parentalité, les traitements disponibles, etc.) ou d'enseigner des stratégies d'adaptation, des consultations, une thérapie familiale serait nécessaire pour celles plus en difficulté (Foster et al., 2012). À cet égard, des études menées auprès de familles comptant un JPTM enfant ou adolescent soulignent que les interventions familiales semblent plus efficaces que celles qui n'impliquent que l'enfant ou que le parent (Piché et al., 2020; Thanhäuser et al., 2017). Une adaptation du programme d'intervention Family Talk, conçu pour une clientèle de JPTM enfants et adolescents et leurs familles, et dont l'efficacité comme l'effectivité ont été validées à de nombreuses reprises à l'étranger (p. ex., Beardslee et al., 2003; 2007; Solantaus et al., 2010), pourrait être réalisée pour une clientèle transitant vers l'âge adulte au Québec. Aux cibles d'intervention prévues dans ce programme (c.-à-d., amélioration de la communication au sein de la famille, partage du vécu de chacun en lien avec le trouble parental et répartition des rôles et responsabilités), hautement pertinentes quel que soit l'âge des JPTM, pourraient s'ajouter un accompagnement familial à la prise d'autonomie ou encore un travail autour de l'identité personnelle et familiale de chacun des membres (p. ex., histoire commune, forces de chacun, projets en commun et personnels, etc.), notamment.

Plus largement, les résultats attestent de l'importance d'une meilleure prise en compte de la famille au sein des services en santé mentale, ce qui concorde avec les directives pancanadiennes qui recommandent d'adopter un système de soins et de programmes d'aide intégrés et coordonnés pour les enfants et familles vivant avec un parent ayant un trouble mental, qui reconnaît l'importance des membres de la famille, les soutient dans leur rôle, répond à leurs besoins et favorise leur implication (MacCourt et al., 2013). À ce jour, il n'existe que peu d'information quant à l'utilisation de telles pratiques centrées sur la famille dans les services en santé mentale québécois, mais les membres des familles dont un parent a un trouble mental rapportent encore recevoir des services fragmentés ou en silo (Carrière et al., 2010).

Malgré l'intérêt des résultats obtenus à l'issue de cette étude, il convient de noter que l'échantillon de celle-ci est restreint, quasi exclusivement composé de jeunes femmes et qu'il ne permet donc pas de statuer sur la représentativité des résultats à l'échelle de la population des JPTM. Des études de plus grande envergure visant à préciser les facteurs et processus les plus déterminants pour la résilience des JPTM en général et de certains sousgroupes en particulier (p. ex., chez les jeunes dont le parent a un trouble anxio-dépressif versus un trouble de la personnalité ou un trouble psychotique; chez les jeunes hommes) au cours de leur transition vers l'âge adulte permettront d'affiner le développement de mesures de soutien efficaces à offrir à cette clientèle.

Pour conclure, cette étude illustre combien il semble essentiel d'agir pour favoriser la santé mentale des JPTM lors de leur transition vers l'âge adulte en prenant appui sur les forces et les capacités des jeunes tout en proposant le soutien et les ressources nécessaires, à différents niveaux et en tenant compte de l'ampleur de leurs besoins, pour les aider à affronter les défis auxquels ils sont confrontés. Tandis que cette approche est recommandée pour soutenir la transition vers l'âge adulte des jeunes en général (Audet et al., 2019; Becquet et Goyette, 2014), cette étude permet de préciser comment la décliner auprès de JPTM de 16 à 25 ans.

\section{Financement}

Ce projet de recherche s'est déroulé en partenariat avec l'organisme communautaire le Réseau Avant de Craquer. Ce dernier a été subventionné par le Conseil de recherches en sciences humaines du Canada (892-2018-2024).

\section{Conflit d'intérêts}

Les auteurs n'ont aucun conflit d'intérêts à déclarer.

\section{Références}

Abraham, K. M. et Stein, C. H. (2013). When mom has a mental illness: Role reversal and psychosocial adjustment among emerging adults. Journal of Clinical Psychology, 696), 600-615. https://doi.org/10.1002/jclp.21950

Ali, L., Ahlström, B. H., Krevers, B. et Skärsäter, I. (2012). Daily life for young adults who care for a person with mental illness: A qualitative study. Journal of Psychiatric and Mental Health Nursing, 197), 610-617. http://doi.org/10.1111/j.1365-2850.2011.01829.x

Arnett, J. J. (2014). Emerging adulthood: The winding road from the late teens through the twenties ( $2^{\mathrm{e}}$ éd.). Oxford University Press. https://doi.org/10.1093/acprof:oso/9780199929382.001.0001

Audet, M., Pauzé, R. et Lepage, J. (2019). Mieux comprendre le soutien social durant la transition à la vie adulte chez des jeunes présentant un profil de vulnérabilité : aperçu des connaissances actuelles. Le magazine des psychoéducateurs : La pratique en 
mouvement, 17, 21-23. https://fr.zone-secure.net/38889/982512/\#page=22

Beardslee, W. R., Gladstone, T. R., Wright, E. J. et Cooper, A. B. (2003). A family-based approach to the prevention of depressive symptoms in children at risk: evidence of parental and child change. Pediatrics, 112(2), e119-e131. http://doi.org/10.1542/peds.112.2.e119

Beardslee, W. R., Wright, E. J., Gladstone, T. R. et Forbes, P. (2007). Long-term effects from a randomized trial of two public health preventive interventions for parental depression. Journal of Family Psychology, 21(4), 703-713. https://doi.org/10.1037/0893-3200.21.4.703

Becquet, V. et Goyette, M. (2014). L'engagement des jeunes en difficulté. Sociétés et jeunesses en difficulté, (14). http://journals.openedition.org/sejed/7828

Bergeron, L., Valla, J. P., Breton, J. J., Gaudet, N., Berthiaume, C., Lambert, J., St-Georges, M. et Smolla, N. (2000). Correlates of mental disorders in the Quebec general population of 6 to 14 year olds. Journal of Abnormal Child Psychology, $28(1), 47-62$. https://doi.org/10.1023/A:1005170017815

Bosch, A., Riebschleger, J. et van Loon, L. (2017). Dutch youth of parents with a mental illness reflect upon their feelings of guilt and shame. International Journal of Mental Health Promotion, 19(3), 159-172. https://doi.org/10.1080/14623730.2017.1315955

Brawer-Sherb, M., Keitel, M., Cunningham, S., Ponterotto, J. G. et Lilliquist, B. (2020). Lived experiences of emerging adult women with single mothers: Exploring responses to perceived maternal depression. Emerging Adulthood. Prépublication. https://doi.org/10.1177/2167696820941958

Burt, K. B. et Paysnick, A. A. (2012). Resilience in the transition to adulthood. Development and Psychopathology, 24(2), 493-505. http://doi.org/10.1017/s0954579412000119

Carrière, M., Clément, M., Tétreault, S., Pépin, G., Fortier, M. et Paquet, S. (2010). Réflexion sur les services aux familles dont la mère souffre d'un trouble de santé mentale. Santé Mentale au Québec, 35(2), 185-208. https://doi.org/10.7202/1000559ar

Cohen-Scali, V. et Guichard, J. (2008). L'identité: perspectives développementales. L'orientation scolaire et professionnelle, (37/3), 321-345. https://doi.org/10.4000/osp.1716

Collin, P., Rahilly, K., Richardson, I. et Third, A. (2011). The benefits of social networking service: Literature review. Cooperative Research Centre for Young People, Technology and Wellbeing. https://researchdirect.westernsydney.edu.au/islandora/object/uws\%3A11520/datastream/PDF/view

Collishaw, S., Hammerton, G., Mahedy, L., Sellers, R., Owen, M. J., Craddock, N., Thapar, A. K., Harold, G. T., Rice, R. et Thapar, A. (2016). Mental health resilience in the adolescent offspring of parents with depression: a prospective longitudinal study. The Lancet Psychiatry, 3(1), 49-57. https://doi.org/10.1016/S2215-0366(15)00358-2

Dam, K. et Hall, E. (2016). Navigating in an unpredictable daily life: A metasynthesis on children's experiences living with a parent with severe mental illness. Scandinavian Journal of Caring Sciences, 30(3), 442-457. http://doi.org/10.1111/scs.12285

Douville, L., Dubé, A., Émery, M. et Normand, A. (2017). La démarche Photovoice à titre d'outil de changement social auprès des jeunes de la rue. Intervention, 145, 43-51. https://revueintervention.org/wpcontent/uploads/2017/05/ri_145_2017.1_douville_et_al.pdf

Ellis, L. et Hoskin, A. (2018). Familial depressive symptoms and delinquency: separate self-reports from mothers and their offspring. International Journal of Offender Therapy and Comparative Criminology, 62(5), 1201-1215. https://doi.org/10.1177/0306624X16678939

Ensminger, M. E., Hanson, S. G., Riley, A. W. et Juon, H. S. (2003). Maternal psychological distress: Adult sons' and daughters' mental health and educational attainment. Journal of the American Academy of Child and Adolescent Psychiatry, 42(9), 1108-1115. https://doi.org/10.1097/01.CHI.0000070261.24125.F8

Foster, K., Goodyear, M., Grant, A., Weimand, B. et Nicholson, J. (2019). Family-focused practice with EASE: A practice framework for strengthening recovery when mental health consumers are parents. International Journal of Mental Health Nursing, 28(1), 351360. https://doi.org/10.1111/inm.12535

Foster, K., O'Brien, L. et Korhonen, T. (2012). Developing resilient children and families when parents have mental illness: A familyfocused approach. International Journal of Mental Health Nursing, 21(1), 3-11. https://doi.org/10.1111/j.14470349.2011.00754.x

Goldstein, A. L., Faulkner, B. et Wekerle, C. (2013). The relationship among internal resilience, smoking, alcohol use, and depression symptoms in emerging adults transitioning out of child welfare. Child Abuse and Neglect, 371), 22-32. https://doi.org/10.1016/j.chiabu.2012.08.007

Gray, N. J., Klein, J. D., Noyce, P. R., Sesselberg, T. S. et Cantrill, J. A. (2005). The Internet: a window on adolescent health literacy. Journal of Adolescent Health, 373), 243-247. https://doi.org/10.1016/j.jadohealth.2004.08.023

Grob, R., Schlesinger, M., Wise, M. et Pandhi, N. (2020). Stumbling into adulthood: Learning from depression while growing up. Qualitative Health Research, 3099), 1392-1408. https://doi.org/10.1177/1049732320914579 
Institute of Medicine and National Research Council. (2015). Investing in the health and well-being of young adults. The National Academies Press. https://doi.org/10.17226/18869

Jacobs, R. H., Talati, A., Wickramaratne, P. et Warner, V. (2015). The influence of paternal and maternal major depressive disorder on offspring psychiatric disorders. Journal of Child and Family Studies, 24(8), 2345-2351. https://doi.org/10.1007/s10826-014$0037-y$

Kaimal, G. et Beardslee, W. R. (2015). The perceived impact of parental depression on the narrative construction of personal identity: Reflections from emerging adults. Narrative Works, 5(1), 40-67. https://journals.lib.unb.ca/index.php/NW/article/view/23784

Leipold, B., Munz, M. et Michéle-Malkowsky, A. (2019). Coping and resilience in the transition to adulthood. Emerging Adulthood, 71), 12-20. http://doi.org/10.1177/2167696817752950

Letourneau, N. L., Fedick, C. B., Willms, J. D., Dennis, C. L., Hegadoren, K. et Stewart, M. J. (2006). Longitudinal study of postpartum depression, maternal-child relationships and children's behaviour to 8 years of age. Dans D. Devore (dir.), Parent-Child Relations: New research (p. 45-63). Nova Science Publishers.

Lewandowski, R. E., Verdeli, H., Wickramaratne, P., Warner, V., Mancini, A. et Weissman, M. (2014). Predictors of positive outcomes in offspring of depressed parents and non-depressed parents across 20 years. Journal of Child and Family Studies, 23(5), 800-811. https://doi.org/10.1007/s10826-013-9732-3

Lorenz, L. S. et Kolb, B. (2009). Involving the public through participatory visual research methods. Health Expectations, 12(3), 262274. https://doi.org/10.1111/j.1369-7625.2009.00560.x

Luecken, L. J. et Gress, J. L. (2010). Early adversity and resilience in emerging adulthood. Dans J. W. Reich, A. J. Zautra et J. S. Hall (dir.), Handbook of Adult Resilience (p. 238-257). The Guilford Press.

MacCourt, P., Comité consultatif sur les aidants membres de la famille et Commission de la santé mentale du Canada. (2013). Directives pancanadiennes en faveur d'un système de prestation de services pour les proches aidants d'adultes ayant une maladie mentale. Commission de la Santé Mentale du Canada. https://www.mentalhealthcommission.ca/Francais/media/3273

Madewell, A. N. et Ponce-Garcia, E. (2016). Assessing resilience in emerging adulthood: The resilience scale (RS), Connor-Davidson resilience scale (CD-RISC), and scale of protective factors (SPF). Personality and Individual differences, 97, $249-255$. https://doi.org/10.1016/j.paid.2016.03.036

Marcotte, J., Villatte, A. et Levesque, G. (2014). La diversité et la complexité des jeunes (16-24 ans) inscrits à l'éducation des adultes : enquête et essai de typologie. Revue des sciences de l'éducation, 40(2), 253-285. https://doi.org/10.7202/1028421ar

Marcotte, J., Villatte, A., Vrakas, G. et Laliberté, A. (2019). L'identité narrative de jeunes dits « vulnérables » s'apprêtant à transiter vers l'âge adulte au Québec. Psychologie française, 64(3), 241-255. https://doi.org/10.1016/j.psfr.2018.01.002

Masten, A. S., Burt, K. B., Roisman, G. I., Obradović, J., Long, J. D. et Tellegen, A. (2004). Resources and resilience in the transition to adulthood: Continuity and change. Development and Psychopathology, 16(4), 1071-1094. https://doi.org/10.1017/S0954579404040143

Masten, A. S., Obradović, J. et Burt, K. B. (2006). Resilience in emerging adulthood: Developmental perspectives on continuity and transformation. Dans J. J. Arnett et J. L. Tanner (dir.), Emerging adults in America: Coming of age in the 21st century (p. 173-190). American Psychological Association.

Metz, D. et Jungbauer, J. (2021). "My scars remain forever": A qualitative study on biographical developments in adult children of parent with mental illness. Clinical Social Work Journal, 49(1), 64-76. https://doi.org/10.1007/s10615-019-00722-2

Ministère de la Santé et des Services Sociaux. (2015). Programme national de santé publique 2015 - 2025. Gouvernement du Québec. http://publications.msss.gouv.qc.ca/msss/fichiers/2015/15-216-01W.pdf

Ministère de la Santé et des Services Sociaux. (2016a). La participation de personnes utilisatrices de services et de membres de l'entourage à la planification et à l'organisation des services: Guide d'accompagnement (Publication nº 16-914-08W). Gouvernement du Québec. http://publications.msss.gouv.qc.ca/msss/fichiers/2016/16-914-08W.pdf

Ministère de la Santé et des Services Sociaux (2016b). La lutte contre la stigmatisation et la discrimination dans le réseau de la santé et des services sociaux - Guide d'accompagnement. Gouvernement du Québec. http://publications.msss.gouv.qc.ca/msss/fichiers/2016/16-914-06W.pdf

Ministère de la Santé et des Services Sociaux. (2017). Plan d'action en santé mentale 2015-2020 - Faire ensemble et autrement. Gouvernement du Québec. https://publications.msss.gouv.qc.ca/msss/fichiers/2017/17-914-17W.pdf

Mordoch, E. et Hall, W. A. (2008). Children's perceptions of living with a parent with a mental illness: Finding the rhythm and maintaining the frame. Qualitative Health Research, 18(8), 1127-1144. https://doi.org/10.1177/1049732308320775

Mottaghipour, Y. et Bickerton, A. (2005). The Pyramid of Family Care: A framework for family involvement with adult mental health services. Australian e-Journal for the Advancement of Mental Health, 4(3), 210-217. https://doi.org/10.5172/jamh.4.3.210

Neziroglu, F., Upston, M. et Khemlani-Patel, S. (2020). The psychological, relational, and social impact in adult offspring of parents 
with hoarding disorder. Children Australia, 45(3), 153-158. https://doi.org/10.1017/cha.2020.42

Ng-Knight, T., Shelton, K. H., Frederickson, N., McManus, I. C. et Rice, F. (2018). Maternal depressive symptoms and adolescent academic attainment: Testing pathways via parenting and self-control. Journal of Adolescence, 62, 61-69. https://doi.org/10.1016/j.adolescence.2017.11.003

Oulasvirta, A., Rattenbury, T., Ma, L. et Raita, E. (2012). Habits make smartphone use more pervasive. Personal Ubiquitous Comput, 16(1), 105-114. https://doi.org/10.1007/s00779-011-0412-2

Pargas, R. C. M., Brennan, A. P., Hammen, C. et Le Brocque, R. (2010). Resilience to maternal depression in young adulthood. Developmental Psychology, 46(4), 805-814. https://doi.org/10.1037/a0019817

Patrick, P., Reupert, A. et McLean, L. (2019). "We are more than our parents' mental illness": Narratives from adult children. International Journal of Environmental Research and Public Health, 16(5), 839. https://doi.org/10.3390/ijerph16050839

Pauzé, R., Lepage, J., Audet, M. et Guy, A. (2019). Développement d'une carte conceptuelle pour mieux accompagner les jeunes vulnérables dans leur transition vers l'âge adulte. La Pratique en Mouvement, 17, 11-13. https://fr.zonesecure.net/38889/982512/\#page=12

Piché, G., Vetri, K., Villatte, A. et Habib, R. (2020). Étude pilote d'une intervention préventive ciblée, pour les enfants et familles vivant avec un parent dépressif. [Manuscrit soumis]. Revue Psychoéducation.

Piché, G., Villatte, A. et Habib, R. (2019). Interventions préventives ciblant les enfants, adolescents et jeunes adultes vivant avec un parent ayant un trouble mental : recension des meilleures pratiques et recommandations. [Manuscrit accepté]. Dans G. Piché, S. Bourque et A. Villatte (dir.), Parentalité et problèmes de santé mentale. Presses de l'Université Laval.

Reupert, A., Bartholomew, C., Cuff, R., Foster, K., Matar, J., Maybery, D. J. et Pettenuzzo, L. (2019). An online intervention to promote mental health and wellbeing for young adults whose parents have mental illness and/or substance use problems: theoretical basis and intervention description. Frontiers in Psychiatry, 10, Article 59. https://doi.org/10.3389/fpsyt.2019.00059

Roberge, M. C. et Déplanche, F. (2017). Knowledge synthesis of relevant spheres of action for promoting the mental health of young adults. Institut National de Santé du Publique Québec. https://www.inspq.qc.ca/sites/default/files/publications/2286_knowledge_relevant_spheres_action_promoting_mental_he alth_young_adults.pdf

Schulenberg, J. E., Bryant, A. L. et O'Malley, P. M. (2004). Taking hold of some kind of life: How developmental tasks relate to trajectories of well-being during the transition to adulthood. Development and Psychopathology, 16(4), 1119-1140. https://doi.org/10.1017/s0954579404040167

Secrétariat à la Jeunesse. (2016). Politique québécoise de la jeunesse 2030: Ensemble pour les générations présentes et futures. Gouvernement du Québec. https://www.jeunes.gouv.qc.ca/publications/documents/pqj-2030.pdf

Siegenthaler, E., Munder, T. et Egger, M. (2012). Effect of preventive interventions in mentally ill parents on the mental health of the offspring: Systematic review and meta-analysis. Journal of the American Academy of Child and Adolescent Psychiatry, 51(1), 8-17. https://doi.org/10.1016/j.jaac.2011.10.018

Simpson-Adkins, G. J. et Daiches, A. (2018). How do children make sense of their parent's mental health difficulties: A metasynthesis. Journal of Child and Family Studies, 27(9), 2705-2716. https://doi.org/10.1007/s10826-018-1112-6

Solantaus, T., Paavonen, E. J., Toikka, S. et Punamäki, R. (2010). Preventive interventions in families with parental depression: children's psychosocial symptoms and prosocial behaviour. European Child and Adolescent Psychiatry, 19(12), 883-892. https://doi.org/10.1007/s00787-010-0135-3

Thanhäuser, M., Lemmer, G., De Girolamo, G. et Christiansen, H. (2017). Do preventive interventions for children of mentally ill parents work? Results of a systematic review and meta-analysis. Current Opinion in Psychiatry, 3044), $283-299$. https://doi.org/10.1097/YCO.0000000000000342

Trondsen, M. V. (2012). Living with a mentally ill parent: exploring adolescents' experiences and perspectives. Qualitative Health Research, 22(2), 174-188. https://doi.org/10.1177/1049732311420736

Van Doesum, K. T. M., Hosman, C. M. H. et Riksen-Walraven, J. M. (2005). A model-based intervention for depressed mothers and their infants. Infant Mental Health Journal, 26(2), 157-176. https://doi.org/10.1002/imhj.20037

van Loon, L. M., van De ven, M. O., van Doesum, K. T., Hosman, C. M. et Witteman, C. L. (2015). Factors promoting mental health of adolescents who have a parent with mental illness: A longitudinal study. Child Youth Care Forum, 44(6), 777-799. https://doi.org/10.1007/s10566-015-9304-3

Van Santvoort, F., Hosman, C. M., Janssens, J. M., Van Doesum, K. T., Reupert, A. et Van Loon, L. M. (2015). The impact of various parental mental disorders on children's diagnoses: A systematic review. Clinical Child and Family Psychology Review, 18(4), $281-299$. http://doi.org/10.1007/s10567-015-0191-9

Villatte, A., Piché, G. et Vetri, K. (2020). L'importance d'intervenir auprès des jeunes ayant un parent atteint d'un trouble mental, de la naissance jusqu'à l'âge adulte. [Mémoire présenté à la Commission des relations avec les citoyens lors des consultations 
particulières sur le Projet de loi no 56 : Loi visant la reconnaissance et le soutien des personnes proches aidantes]. Montréal.

Wang, C. et Burris, M. A. (1997). Photovoice: Concept, methodology, and use for participatory needs assessment. Health Education and Behavior, 24(3), 369-387. https://doi.org/10.1177/109019819702400309

Weich, S., Brugha, T., King, M., McManus, S., Bebbington, P., Jenkins, R., Cooper, C., McBride, O. et Stewart-Brown, S. (2011). Mental well-being and mental illness: Findings from the adult psychiatric morbidity survey for England 2007. British Journal of Psychiatry, 199(1), 23-28. http://doi.org/10.1192/bjp.bp.111.091496

Weissman, M., Wickramaratne, P., Nomura, Y., Warner, V., Pilowsky, D. et Verdeli, H. (2006). Offspring of depressed parents: 20 years later. American Journal of Psychiatry, 163(6), 1001-1008. http://doi.org/10.1176/ajp.2006.163.6.1001

Yamamoto, R. et Keogh, B. (2018). Children's experiences of living with a parent with mental illness: A systematic review of qualitative studies using thematic analysis. Journal of Psychiatric and Mental Health Nursing, 25(2), 131-141. http://doi.org/10.1111/jpm.12415 